\title{
Mass diffusion of organic fluids: a molecular dynamics perspective
}

Alex Smolyanitsky Andrei F. Kazakov

Thomas J. Bruno

Marcia L. Huber 


\title{
Mass diffusion of organic fluids: a molecular dynamics perspective
}

\author{
Alex Smolyanitsky \\ Andrei F. Kazakov \\ Thomas J. Bruno \\ Marcia L. Huber \\ Applied Chemicals and Materials Division \\ Materials Measurement Laboratory
}

http://dx.doi.org/10.6028/NIST.TN.1805

May 2013

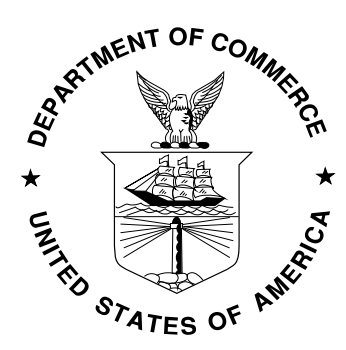

U.S. Department of Commerce Rebecca Blank, Acting Secretary

National Institute of Standards and Technology Patrick D. Gallagher, Under Secretary of Commerce for Standards and Technology and Director 
Certain commercial entities, equipment, or materials may be identified in this document in order to describe an experimental procedure or concept adequately. Such identification is not intended to imply recommendation or endorsement by the National Institute of Standards and Technology, nor is it intended to imply that the entities, materials, or equipment are necessarily the best available for the purpose.

National Institute of Standards and Technology Technical Note 1805

Natl. Inst. Stand. Technol. Tech. Note 1805, 16 pages (May 2013) CODEN: NTNOEF

http://dx.doi.org/10.6028/NIST.TN.1805 


\title{
Mass diffusion of organic fluids: a molecular dynamics perspective
}

\begin{abstract}
The Optimized Potential for Liquid Simulation All-Atom (OPLS-AA) forcefield, a wellestablished interaction potential, within the MD framework was used to determine self- and mutual diffusivity of several fluids, in the high-pressure and near- and supercritical regimes. The test fluids were benzene, heptane and carbon dioxide. The Green-Kubo method of calculating mass diffusivity yielded considerably overestimated values of self-diffusivity; better results were obtained with use of the Stokes-Einstein method. In addition, a number of relatively significant problems with the default OPLS-AA parameterization were identified. There exist ranges of pressure and temperatures, in which the results for the selected pure fluids show good agreement with experiment, and thus reparameterization of OPLS-AA may not be required. However, the results for mixtures all show significant deviation from experiment. Based on the observed results, the current predictive power of OPLS-AA for mass diffusivity of the fluids in question is not satisfactory. We are therefore convinced that a systematic reparameterization of OPLS-AA both in terms of the intermolecular (van der Waals, electrostatic) and intramolecular (bonding) interactions is needed to increase its accuracy. In addition, OPLS-AA may not be generally appropriate for the gaseous state and therefore near-critical systems may benefit from using a different forcefield.
\end{abstract}




\section{Introduction}

Knowledge of the fluid transport properties is crucial for our understanding of the way a pure fluid or a mixture responds to changes in pressure, or temperature. Of great importance in the design and optimization of mass transfer processes is the diffusion coefficient. A recent application of particular interest is the concept of clean diesel combustion that incorporates the use of supercritical carbon dioxide to reduce emissions and improve engine efficiency. Accurate values of the diffusion coefficients of hydrocarbons in supercritical carbon dioxide are essential to the understanding and optimal design of this process.

Determining fluid diffusion properties in the supercritical regime is challenging from the experimental standpoint [1-4], which often reduces the practicality of direct measurement. Current analytical calculations based on kinetic theory can provide an adequate description of the self-diffusivity of pure fluids, but yield significantly less

accurate results for mixtures [5]. As a result, molecular dynamics (MD) simulations have been utilized to determine the mutual mass diffusivity of mixtures (as well as pure fluids) with relative success [5-7].

The aim of this investigation is to determine the feasibility of using one of the most established interaction potentials, the Optimized Potential for Liquid Simulation AllAtom (OPLS-AA) force-field [8] within the MD framework, to determine self- and mutual diffusivity of near-critical and supercritical carbon dioxide, as well as organic fuels, at high pressure. For test fluids we chose benzene, heptane, and carbon dioxide due 
to their relevance as representative fluids for clean diesel combustion as well as the availability of some experimental data to validate our calculations. We report the calculated mass density and diffusion data obtained with OPLS-AA, as a function of pressure and temperature. We discuss the current performance of OPLS-AA in terms of accuracy of calculations, as well as possible modifications necessary to obtain better agreement with known experimental data, and therefore increase the general predictive power of the MD-based approach.

\section{Description of the simulated system}

The fluid systems simulated here represented carbon dioxide, benzene, and heptane; each pure fluid system consisted of a total of 4000 molecules, unless stated otherwise. All mixtures studied here contained 4000 molecules of solvent and 40 molecules of solute, in order to mimic conditions close to infinite dilution. The simulations were performed with use of the GROMACS 4.5.6 package [9] configured with double precision. The pressures and temperatures were appropriately maintained by baro- and thermostatics, respectively [9]. Each system was pre-relaxed with temperature and pressure control in a two-step procedure for a total of 1 ns, followed by a production simulation. The production simulations of pure fluids were performed at constant volume and temperature and run for a total of $5 \mathrm{~ns}$; simulations of mixtures were run for 15 ns. The simulated time intervals were selected to ensure overall numerical convergence and thus statistical significance of the calculated properties. All statistical averages were calculated in the production simulations during the second half of the simulated time interval. 
The interatomic interactions for heptane and benzene were described by the corresponding default OPLS-AA models [8]. Carbon dioxide was represented by a rigid linear system with two virtual atomic sites of appropriate mass, two oxygen atoms, and one carbon atom. All atoms were fully represented in terms of the van der Waals and electrostatic interactions [9]. The electrostatic interactions were calculated from the particle-particle sum and a long-range particle-mesh correction obtained from the appropriate Ewald sum [9]. The van der Waals and short-range electrostatic cut-off radius used here was $1 \mathrm{~nm}$. Within the OPLS-AA formulation, the non-bonded short-range van der Waals interactions were represented by the Lennard-Jones pairwise potential:

$\varphi(r)=4 \epsilon\left[\left(\frac{\sigma}{r}\right)^{12}-\left(\frac{\sigma}{r}\right)^{6}\right]$

where $\epsilon$ and $\sigma$ are the depth and position of the pairwise energy well. The Lennard-Jones parameters along with the partial electric charges $q$ are provided in Table 1 , and the interspecies mixing rules were implemented according to OPLS-AA, as follows: $\sigma_{A B}=$ $\sqrt{\sigma_{A} \sigma_{B}} \cdot \epsilon_{A B}=\sqrt{\epsilon_{A} \epsilon_{B}}$

\begin{tabular}{|l|l|l|l|}
\hline & $\boldsymbol{\epsilon}(\mathbf{K})$ & $\boldsymbol{\sigma}(\AA)$ & $\mathbf{Q}(|\mathbf{e}|)$ \\
\hline $\begin{array}{l}\text { Carbon dioxide } \\
\left(\mathrm{CO}_{2}\right)\end{array}$ & C-C: 28.13 & C-C: 2.76 & C: +0.652 \\
\hline Benzene $\left(\mathrm{C}_{6} \mathrm{H}_{6}\right)$ & O-O: 80.51 & O-O: 3.03 & H: -0.326 \\
& C-C: 35.24 & C-C: 3.55 & C: +0.115 \\
Heptane $\left(\mathrm{C}_{7} \mathrm{H}_{16}\right)$ & H-H: 15.10 & H-H: 2.42 & H: -0.115 \\
& H-H: $: 15.23$ & C-C: 3.50 & C (terminus): -0.18 \\
& & H-H: 2.50 & C (backbone): -0.12 \\
& & H: +0.06 \\
\hline
\end{tabular}

Table 1. Lennard-Jones parameters and partial electric charges used in the simulations. The carbon dioxide parameters are from literature [10, 11], while the parameters for benzene and heptane are the OPLS-AA's defaults for the corresponding compound. 
The mass density was calculated directly from the ratio of the total mass of the system to its volume. The self-diffusion coefficients for the pure fluids were calculated with use of the Stokes-Einstein relation:

$D_{A}=\left.\frac{1}{6 \tau}\left\langle\sum \Delta \boldsymbol{r}^{2}\right\rangle\right|_{\tau}$,

where $\Delta \boldsymbol{r}$ is the molecular displacement and the averaging is calculated for all molecules of species $\boldsymbol{A}$, integrated over the period $\tau$. For mixtures, the corresponding expression is more complex and is provided elsewhere [6]. However, for the case of infinite dilution, the expression for mutual mass diffusivity conveniently reduces to the solute's selfdiffusivity. Note that for numerical comparison in some cases, we also calculated the diffusion coefficients with use of the Green-Kubo method [5].

\section{Results}

\section{Carbon dioxide}

Shown in Fig. 1 is the carbon dioxide mass density data obtained for various temperatures, compared with the NIST Refprop [12] data. Good agreement is obtained for the sub-critical regime $(<50 \mathrm{bar})$ at $\mathrm{T}=298 \mathrm{~K}$, while a significant discrepancy $(25 \%)$ is observed for the supercritical point at $\approx 140 \mathrm{bar}$. For the temperatures of $323 \mathrm{~K}$ and 348 $\mathrm{K}$, the agreement between Refprop data and the $\mathrm{MD}$ results also decreases with increasing pressure. The observed discrepancies strongly suggest that the compressibility of carbon dioxide with the current van der Waals and electrostatic interaction parameters is tailored within default OPLS-AA toward lower pressures simulated here. 

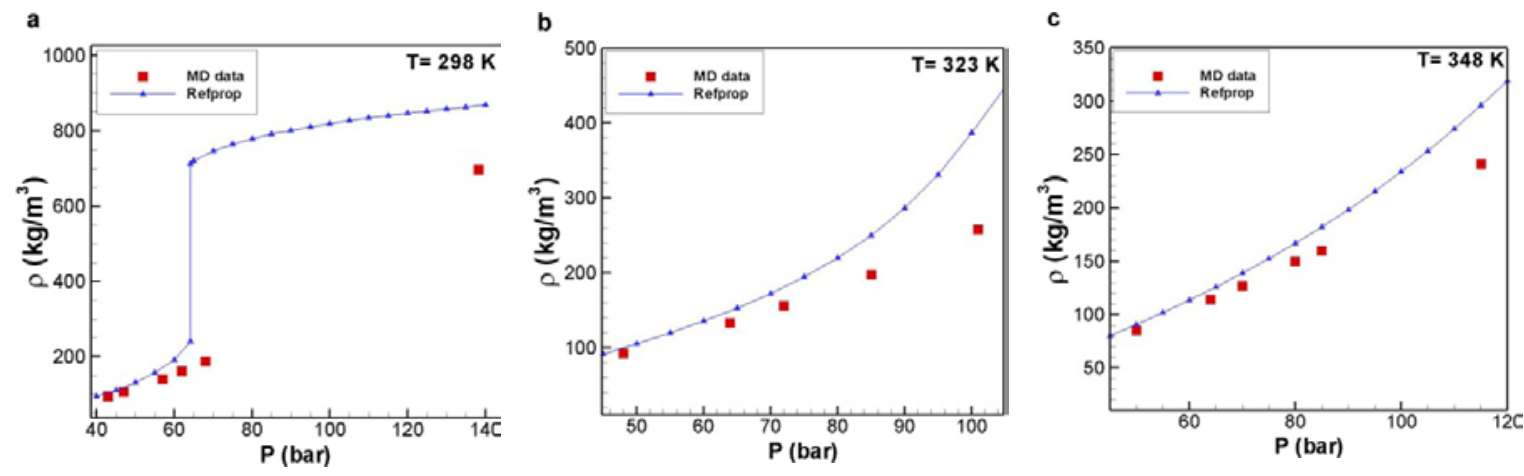

Figure 1. Carbon dioxide mass density results obtained with OPLS-AA force-field for the temperatures of 298 K (a), 323 K (b), and 348 K (c), compared with NIST Refprop data. As we see further, the effect of incorrect compressibility on the carbon dioxide's selfdiffusivity is not significant. Shown in Fig. 2 is the mass self-diffusivity obtained for the same set of temperatures, compared with experimental data [4].
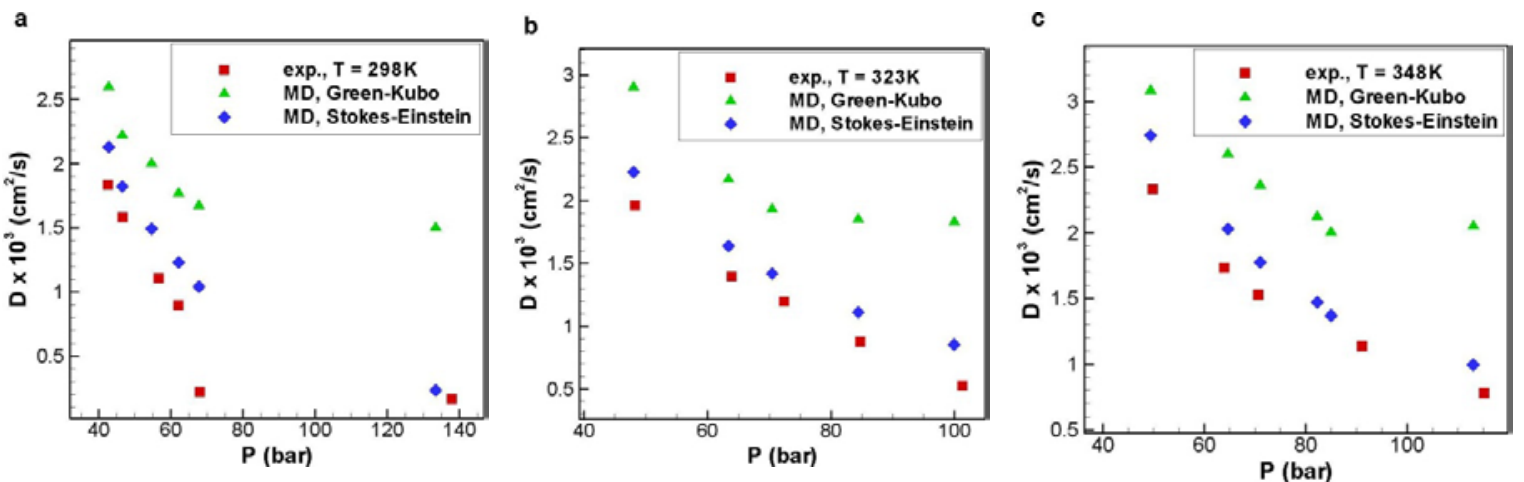

Figure 2. Carbon dioxide mass self-diffusivity obtained with OPLS-AA force-field for the temperatures of $298 \mathrm{~K}$ (a), $323 \mathrm{~K}$ (b), and $348 \mathrm{~K}$ (c), compared with experimental data [4].

As we can see from Fig. 2, the self-diffusivity results obtained with the Stokes-Einstein expression are in good agreement with the experimental data for all temperatures, indicating that an incorrect compressibility at high pressures does not significantly affect the simulated mass diffusion for the carbon dioxide molecule. Moreover, the agreement with experiment is considerably better, compared with other interatomic potentials [13]. 
For all temperatures in Fig. 2, the Green-Kubo method yields considerably overestimated values of self-diffusivity. Such discrepancies, resulting from general numerical convergence issues, are not surprising and have been reported previously [14]. Because of this discrepancy, the diffusivities further in the text were only calculated with use of Stokes-Einstein method.

\section{Benzene}

Shown in Fig. 3 is the benzene mass density data obtained for the temperatures of $298 \mathrm{~K}$ and $328 \mathrm{~K}$, compared with the NIST Refprop [12] data. We see that for T = $298 \mathrm{~K}$ the simulated density agrees with Refprop within $5 \%$. For T $=328 \mathrm{~K}$, the difference between MD and Refprop at low pressures is approximately $6 \%$, improving somewhat toward higher pressures. Such behavior may indicate a slight offset in the van der Waals, as well as electrostatic parameters of OPLS-AA; also, the internal rigidity of each benzene ring may be overestimated, which affects intermolecular packing.
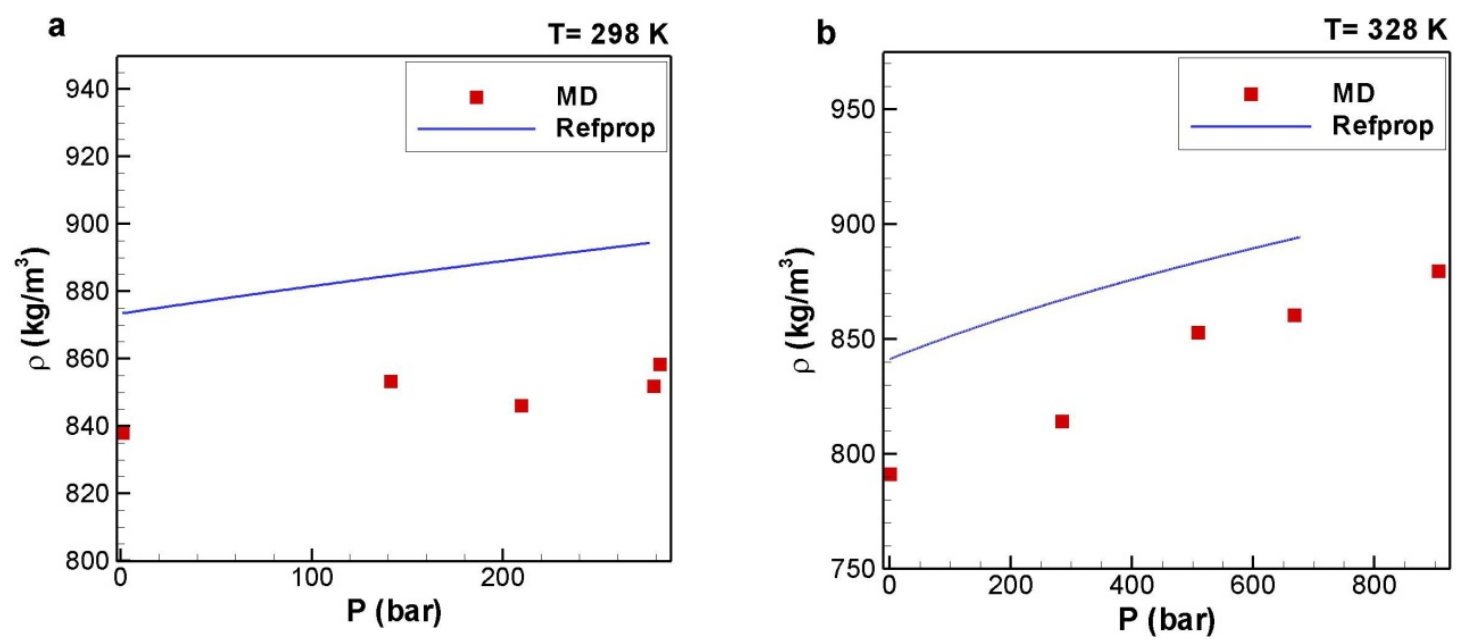

Figure 3. Benzene mass density results obtained with OPLS-AA force-field for the temperatures of 298 K (a), 328 K (b), compared with NIST Refprop data. 
In Fig. 4 we report the benzene self-diffusion results, obtained for the same set of temperatures, compared with experimental data [1]. For $\mathrm{T}=298 \mathrm{~K}$, the average discrepancy with experiment is $20 \%$, while for $\mathrm{T}=328 \mathrm{~K}$, the discrepancy steadily decreases from $25 \%$ to $3 \%$ at the lowest and the highest pressure, respectively.
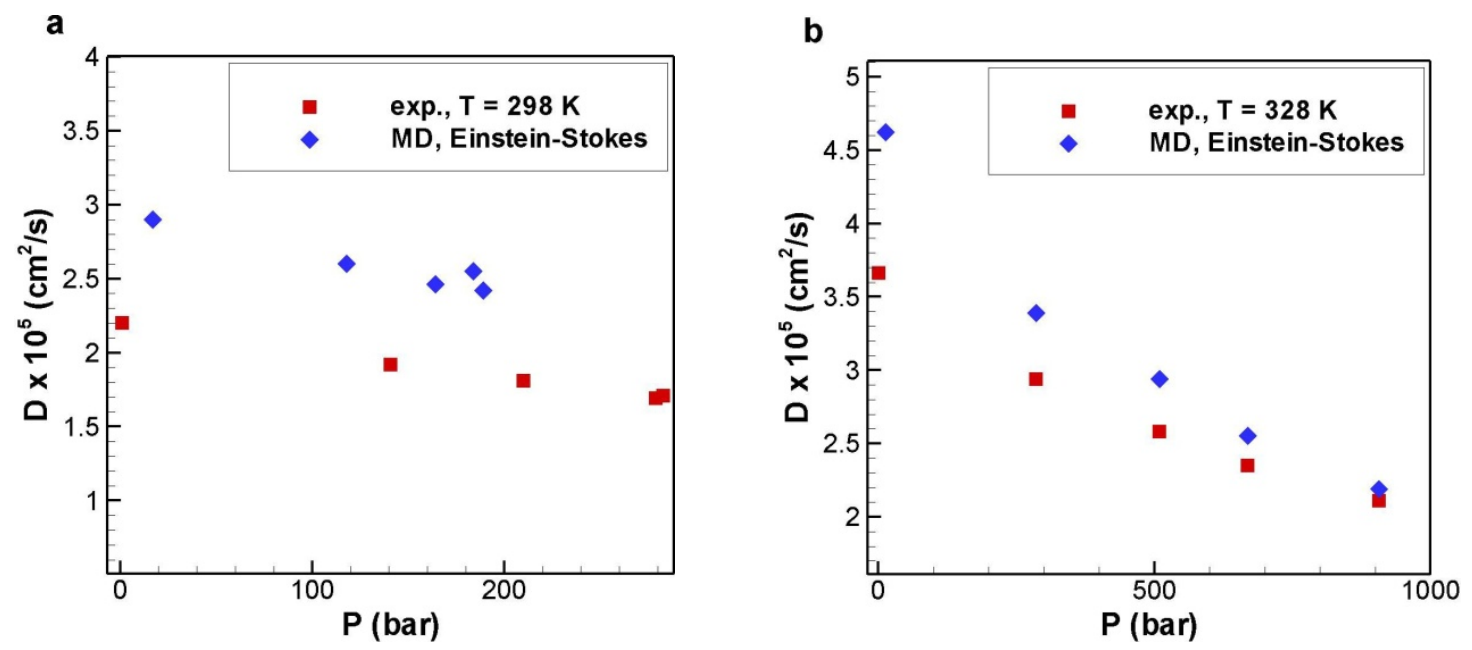

Figure 4. Benzene mass self-diffusivity obtained with OPLS-AA force-field for the temperatures of $298 \mathrm{~K}$ (a) and $328 \mathrm{~K}$ (b), compared with experimental data [1].

This result indicates that for the model of benzene, both the compressibility and mass diffusivity are affected by a relatively severe lack of proper parameterization in OPLSAA. It also appears that the discrepancy between calculated and experimentally obtained self-diffusivity decreases strongly with increasing pressure.

\section{Heptane}

Shown in Fig. 5 are the results for mass density obtained for pure heptane at temperatures of $293 \mathrm{~K}$ and $353 \mathrm{~K}$. The results show considerable discrepancy from Refprop and experimental data at lower pressures, with $10 \%$ to $5 \%$ deviation at $\mathrm{P}=1$ bar for $353 \mathrm{~K}$ and $293 \mathrm{~K}$, respectively. Toward higher pressures, the agreement improves and is on 
average $2 \%$ to $3 \%$. These results suggest that the default OPLS-AA parameterization for heptane (at the temperatures considered) is tailored toward pressures significantly above atmospheric.

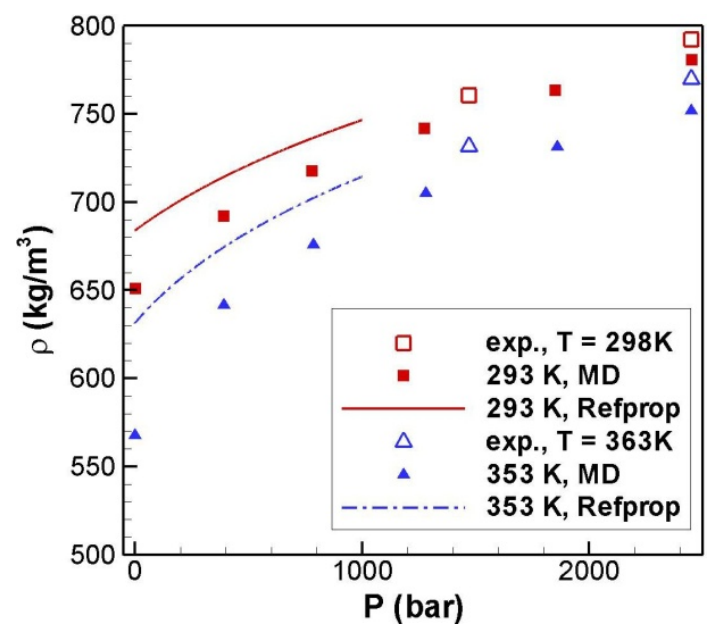

Figure 5. Heptane mass density results obtained with OPLS-AA force-field for the temperatures of $298 \mathrm{~K}$ (a) and $353 \mathrm{~K}$ (b), compared with NIST Refprop data and experimental values [15].

Shown in Fig. 6 are the self-diffusivity results for heptane, compared with experimental data [16]. Similar to the results for benzene in Fig. 4 (b), for both temperatures considered, the discrepancy with experiment decreases from $25 \%$ at the atmospheric pressure to $2 \%$ at the highest pressure of $\approx 2450$ bar. It appears that for the highly flexible heptane molecule, the default OPLS-AA representation provides an excess of bending and rotational degrees of freedom, resulting in overestimated self-diffusivity. It is therefore not surprising that at high pressures these shortcomings of the OPLS-AA default parameterization become negligible and the self-diffusivity approaches experimental values. A comprehensive reparameterization of OPLS-AA energies associated with molecule bending and rotation may solve the observed issue. At the same 
time, if one is interested in the self-diffusivity of heptane for pressures above 1000 bar, the current OPLS-AA performance is already within $10 \%$ of the experimental data.
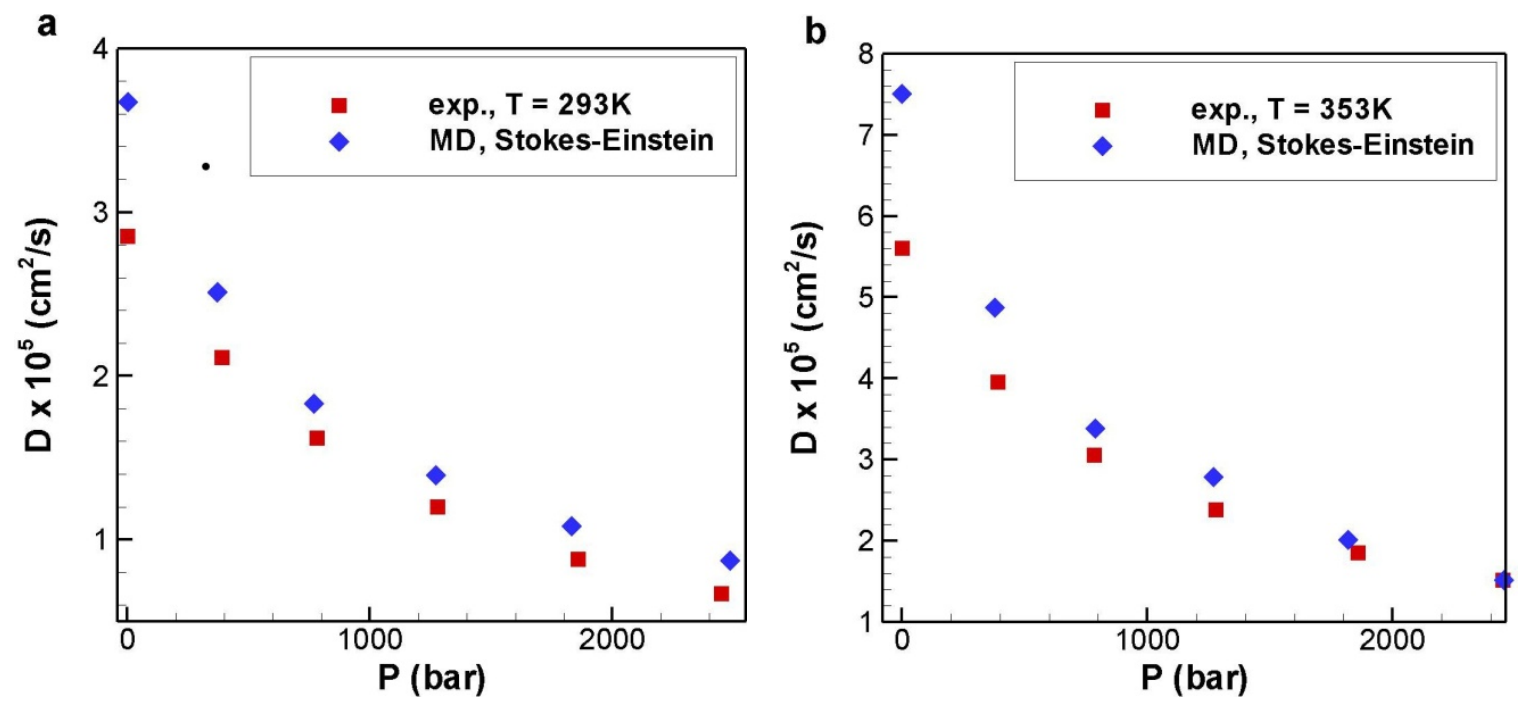

Figure 6. Heptane mass self-diffusivity obtained with OPLS-AA force-field for the temperatures of $293 \mathrm{~K}$ (a) and $353 \mathrm{~K}$ (b), compared with experimental data [16].

\section{Binary mixtures}

Shown in Fig. 7 are the mutual diffusivity data of benzene and heptane infinitely diluted in supercritical carbon dioxide, compared with available experimental data. The relatively narrow range of pressures selected for simulations was due to the fact that the density of carbon dioxide, which dominates the environment of the solute in this case, agreed with experiment within $15 \%$ only for pressure values below 120 bar (Fig. 1). As we can see from Fig. 7, despite the carefully selected range of simulated pressures, the mutual diffusivity for both benzene and heptanes is considerably overestimated. 

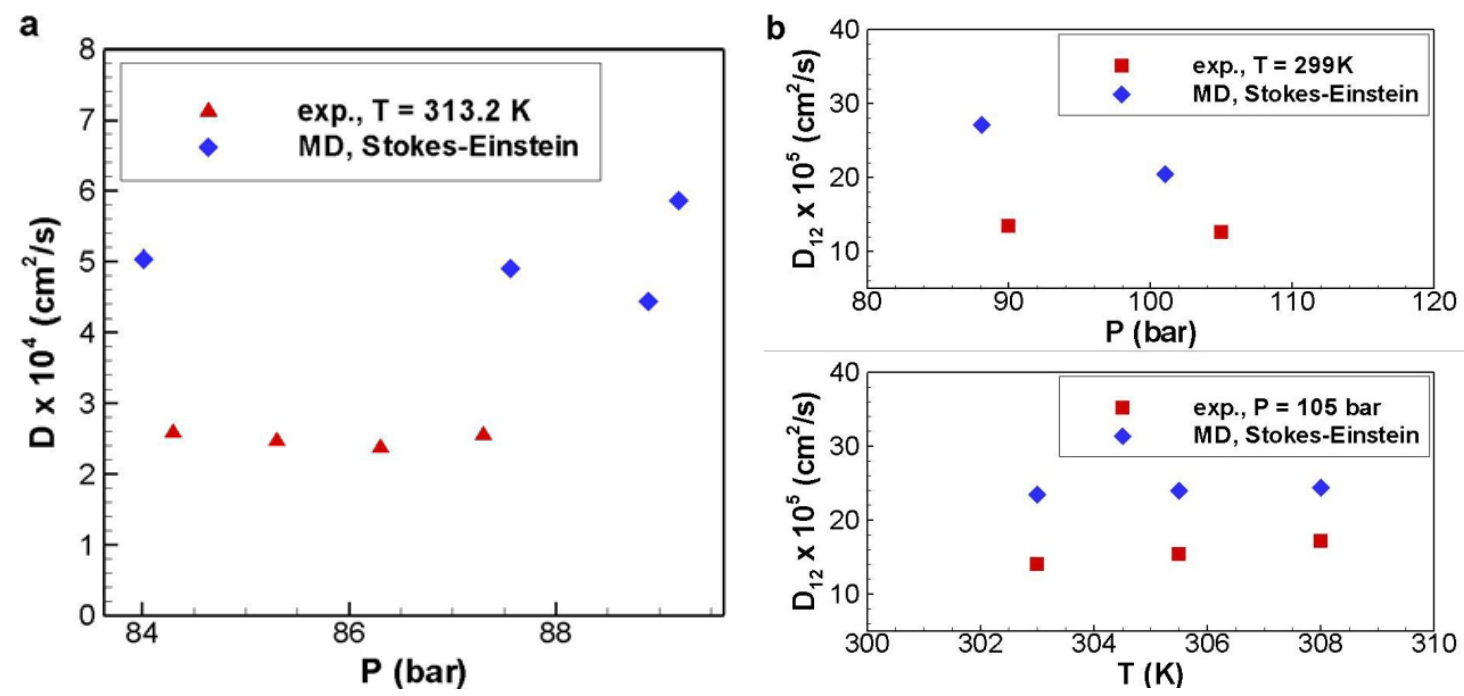

Figure 7. Mutual diffusivity obtained with OPLS-AA force-field for benzene (a) and heptane (b) infinitely diluted in supercritical carbon dioxide. The experimental data for benzene and heptane are from [17] and [18], respectively.

We believe the discrepancy observed here is due to a combination of the underestimated density of the solvent, together with incorrectly described internal degrees of freedom of the solute molecules, which results in greatly overestimated values of mutual diffusivity. While the same phenomenon does not strongly affect the self-diffusivity of carbon dioxide (although its self-diffusivity was also somewhat overestimated; see Fig. 2), the effect for more complex (in terms of the number of degrees of freedom) solute molecule, such as benzene and heptane is significant, resulting in overestimation of mutual diffusivity by at least $100 \%$, compared to experiment. For isotherms at higher pressures, we expect the discrepancy to grow further.

\section{Conclusions, recommendations, and future work}

In this study, we performed a variety of MD simulations with use of OPLS-AA forcefield and reported on the accuracy of density and mass diffusivity for pure near-critical and 
supercritical fluid carbon dioxide, high-pressure benzene, and heptane, as well as (carbon dioxide - benzene) and (carbon dioxide - heptane) mixtures.

A number of significant problems with the default OPLS-AA parameterization were identified. For carbon dioxide, the mass density is strongly underestimated at higher pressures, indicating an incorrect high-pressure compressibility. At the same time, the self-diffusivity of carbon dioxide is calculated within $5 \%$ to $10 \%$ of the established experimental data, which indicates that the issues with compressibility here do not significantly affect self-diffusion. For benzene and heptane, it was observed that the mass densities are consistently underestimated at pressures close to atmospheric (reaching a discrepancy with experiment of at least $\approx 6 \%$ ), while the self-diffusion data is overestimated at lower pressures, compared to experiment. We believe that the observed discrepancies arise from an incorrect default OPLS-AA parameterization; thus, a systematic reparameterization could be of great value. For mixtures, we observe a definite combined effect, in which the mutual diffusivities are overestimated by as high as $100 \%$. We believe that is due to an incorrect compressibility of carbon dioxide in OPLS-AA, combined with the lack of accuracy of OPLS-AA in describing the internal degrees of freedom for benzene and heptane. Finally, because OPLS-AA is designed for the simulation of liquids, it may not be generally appropriate for simulating systems in the selected states, and thus other molecular dynamics forcefields could be used.

Given these observations, great care must be exercised when simulating self- and mutual diffusivity of organic fuels, using the default OPLS-AA parameterization. There exist ranges of pressure and temperatures, in which the results for pure fluids show good 
agreement with experiment, and thus reparameterization of OPLS-AA may not be required (as seen, for example, for heptane in Fig. 6). However, all results for mixtures show significant deviation from experiment. Based on the observed results, the current predictive power of OPLS-AA for mass diffusivity of organic fluids in selected states is not satisfactory. We therefore recommend that a systematic reparameterization of OPLSAA, both in terms of the intermolecular (van der Waals, electrostatic) and intramolecular (bonding) interactions, should be undertaken in order to increase the accuracy of OPLSAA representation of fluid transport properties calculated within the molecular dynamics framework.

\section{References}

1. McCool, M.A., A.F. Collings, and L.A. Woolf, Pressure and temperature dependence of the self-diffusion of benzene. J. Chem. Soc., Faraday Trans., 1972. 1(68): p. 1489-1497.

2. Polzin, B. and A. Weiss, Transport Properties of Liquids. VIII. Molar Volume and Selfdiffusion of Organic Liquids at Pressures up to $200 \mathrm{MPa}$. Berichte der Bunsengesellschaft für physikalische Chemie, 1990. 94(7): p. 746-758.

3. Moore, J.W. and R.M. Wellek, Diffusion Coefficients of n-Heptane and n-Decane in n-Alkanes and n-Alcohols at Several Temperatures. J. Chem. Eng. Data, 1974. 19(2): p. 136-140.

4. $\quad$ Etesse, P., J.A. Zega, and R. Kobayashi, High pressure nuclear magnetic resonance measurement of spin-lattice relaxation and self-diffusion in carbon dioxide. Journal of Chemical Physics, 1992. 97(3): p. 2022-2029.

5. Chae, K. and A. Violi, Mutual diffusion coefficients of heptane isomers in nitrogen: A molecular dynamics study. Journal of Chemical Physics, 2011. 134.

6. Zhang, L., Y.-C. Liu, and Q. Wang, Molecular dynamics simulation of self- and mutual diffusion coefficients for confined mixtures. Journal of Chemical Physics, 2005. 123.

7. Zabaloy, M.S., V.R. Vasquez, and E.A. Macedo, Description of self-diffusion coefficients of gases, liquids and fluids at high pressure based on molecular simulation data. Fluid Phase Equilibria, 2006. 242: p. 43-56.

8. Jorgensen, W.L., D.S. Maxwell, and J. Tirado-Rives, Development and Testing of the OPLS All-Atom Force Field on Conformational Energetics and Properties of Organic Liquids. J. Am. Chem. Soc., 1996. 118(45): p. 11225-11236.

9. Apol, E., et al., GROMACS 4.5.6 manual, 2010. 
10. Chatzis, G. and J. Samios, Binary mixtures of supercritical carbon dioxide with methanol. A molecular dynamics simulation study. Chemical Physics Letters, 2003. 374(1): p. 187-193.

11. Yoo, J.-H., et al., Diffusion coefficients of supercritical carbon dioxide and its mixtures using molecular dynamic simulations. Korean Journal of Chemical Engineering, 2012. 29(7): p. 935-940.

12. Lemmon, E.W., M.L. Huber, and M.O. McLinden, REFPROP, NIST Reference Fluid Thermodynamic and Transport Properties Database 23, Version 9.0, 2010, National Institute of Standards and Technology: Gaithersburg, MD.

13. Coelho, L.A.F., J.V. Oliveira, and F.W. Tavares, Dense fluid self-diffusion coefficient calculations using perturbation theory and molecular dynamics. Brazilian Journal of Chemical Engineering, 1999. 16(3).

14. Wei-Zhong, L., C. Cong, and Y. Jian, Molecular Dynamics Simulation of SelfDiffusion Coefficient and Its Relation with Temperature Using Simple LennardJones Potential. Heat Transfer - Asian Research, 2008. 37(2): p. 86-93.

15. Sagdeev, D.I., et al., Experimental Study of the Density and Viscosity of nHeptane at Temperatures from $298 \mathrm{~K}$ to $470 \mathrm{~K}$ and Pressure up to $245 \mathrm{MPa}$. International Journal of Thermophysics, 2013. 34(1): p. 1-33.

16. Arkhipov, V.P., Experimental study of the diffusion coefficient in liquid nparaffins at high pressures. Izvestiia vysshikh uchebnykh zavedenii. Neft' i gaz, 1982: p. 34-44.

17. Funazukuri, T. and N. Nishimoto, Tracer diffusion coefficients of benzene in dense CO2 at $313.2 \mathrm{~K}$ and 8.5-30MPa. Fluid Phase Equilibria, 1996. 125(1-2): p. 235-243.

18. Umezawa, S. and A. Nagashima, Measurement of the Diffusion Coefficients of Acetone, Benzene, and Alkane in Supercritical CO2 by the Taylor Dispersion Method. The Journal of Supercritical Fluids, 1992. 5(4): p. 242-250. 